\title{
Fully Endoscopic Transforaminal Discectomy under Local Anesthesia for Thoracic Disc Herniations: A Case Series
}

\author{
Jian Shen*, MD, PhD
}

Department of Spine Surgery, Mohawk Valley Orthopedics, Center for Spine Regeneration Surgery, New York, USA

\begin{abstract}
Background: Symptomatic thoracic disc herniation is a relatively rare yet challenging-to-treat condition. Minimally invasive approaches potentially offer less complications and quick recovery.

Purpose: Here we describe a step-by-step technique for fully-endoscopic foraminoplasty with high-speed drill under direct visualization through transforaminal/retropleural approach, followed by discectomy to treat thoracic disc herniations under local anesthesia, and report the primary results of a case series.

Results: Between January 2012 and December, 2017, 16 patients with symptomatic thoracic disc herniation were treated with fully endoscopic transforaminal foraminoplasty and discectomy under local anesthesia. Intraoperative data and clinical outcomes were analyzed and reported. One patient with a chronic calcified giant central disc herniation and significant myelopathy had complaints of worsening symptoms postoperatively; she subsequently underwent open multi-level laminectomy fusion surgery elsewhere. For the remaining 15 patients, at the final follow up (mean: 21 months; range: 7-60 months), the mean VAS was improved from 7.9 to 2.1. No other complications were observed or reported during and after the surgery.
\end{abstract}

Conclusion: Results from this case series shows that fully-endoscopic transforaminal/retropleural foraminoplasty and discectomy under local anesthesia is a safe and effective treatment for symptomatic soft and calcified thoracic disc herniations.

Keywords: Thoracic disc herniation; Fully endoscopic; Transforaminal/ retropleural approach; Foraminoplasty under direct visualization

\section{Introduction}

Thoracic disc herniations are rare, constitute only $0.15 \%$ to $4 \%$ of all spinal discectomy surgeries [1]. Intolerable and persistent pain that failed conservative treatments or acute or progressive neurological deficit is relative indications for surgical treatments, which can be challenging. Individual planning is required and various surgical techniques and approaches have been used [2-4]. In decompression surgery, manipulation or retraction of the spinal cord must be avoided. Endoscopic uniportal technique through transforaminal/retropleural approach under continuous irrigation has been reported with good outcomes and advantages $[5,6]$. In the transforaminal approach, the foramen has to be widened with bone resection of the superior facet and part of the rib head. This case series describes a step-by-step technique for fully-endoscopic foraminoplasty with high-speed drill under direct visualization, followed by discectomy to treat thoracic disc herniations, and its unique advantages to achieve adequate decompression with uniportal transforaminal/retropleural approach under local anesthesia.

\section{Materials and Methods}

\section{Operative procedure}

Under local anesthesia with IV sedation, the surgery was performed with patient in prone position on radiolucent table, under biplanar fluoroscopic guidance. The level of anesthetic was titrated so the patient was comfortable and able to communicate with the surgeon throughout the surgery (Figures 1A and 1B). Preoperatively, the approach angle was measured medial to the ribs and pleura and the incision was planned. Percutaneous entry was established entering through the skin $7-9 \mathrm{~cm}$ lateral to the midline. Using intermittent fluoroscopic guidance, alternating between lateral and anterior-posterior (AP) views, a 25$\mathrm{cm} 18$-gauge needle was advanced and placed at the superior facet of the surgical level (Figures 2A and 2B). Subsequently, the dilator and the working sheath was placed bluntly through the soft tissue and docked on the ipsilateral superior facet (Figures 2C and 2D). The
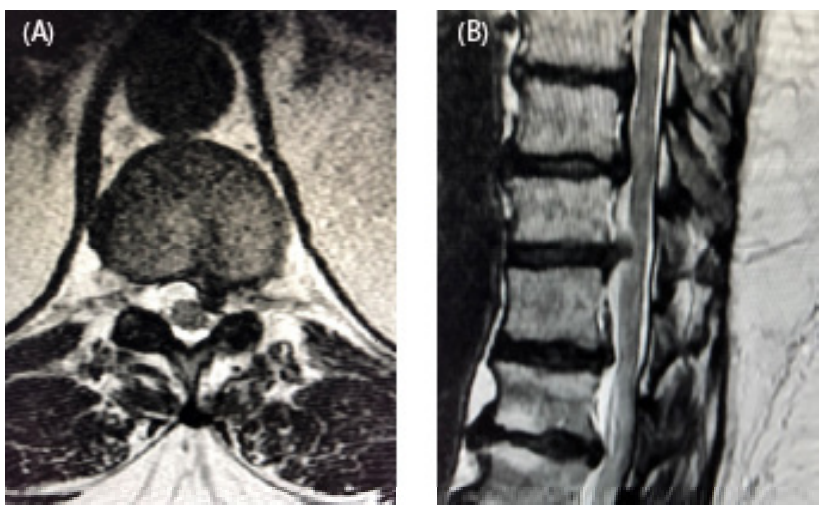

Figures 1A and 1B: MRI images of a 56-year-old female patient with a symptomatic T11/T12 left paracentral disc herniation.

Joimax (Irvine, USA) TESSYS endoscopic system with a 25-degree viewing angle was used. All surgical instruments were introduced under continuous irrigation and direct visualization through the intraendoscopic working channel. Thoracic foramen is enclosed by facet, pedicle and rib head. Foraminoplasty was done with high-speed drill (Joimax Shrill, $3.5 \mathrm{~mm}$ diamond abrasor) to enlarge the neural foramen by drilling the ventral aspect of the superior facet (Figures $3 \mathrm{~A}$ and $3 \mathrm{~B}$ )

*Corresponding author: Jian Shen, Department of Spine Surgery, Mohawk Valley Orthopedics, 5010 State Highway 30, Amsterdam, NY 12010; Center for Spine Regeneration Surgery, 215 East 77th Street, New York, NY 10075, USA, Tel: + 518842-2663, E-mail: james2173@yahoo.com

Received August 24, 2018; Accepted September 03, 2018; Published September 06, 2018

Citation: Shen J (2018) Fully Endoscopic Transforaminal Discectomy under Local Anesthesia for Thoracic Disc Herniations: A Case Series. J Spine S7: 013. doi: 10.0132/2165-7939.S7-013

Copyright: @ 2018 Shen J. This is an open-access article distributed under the terms of the Creative Commons Attribution License, which permits unrestricted use, distribution, and reproduction in any medium, provided the original author and source are credited. 

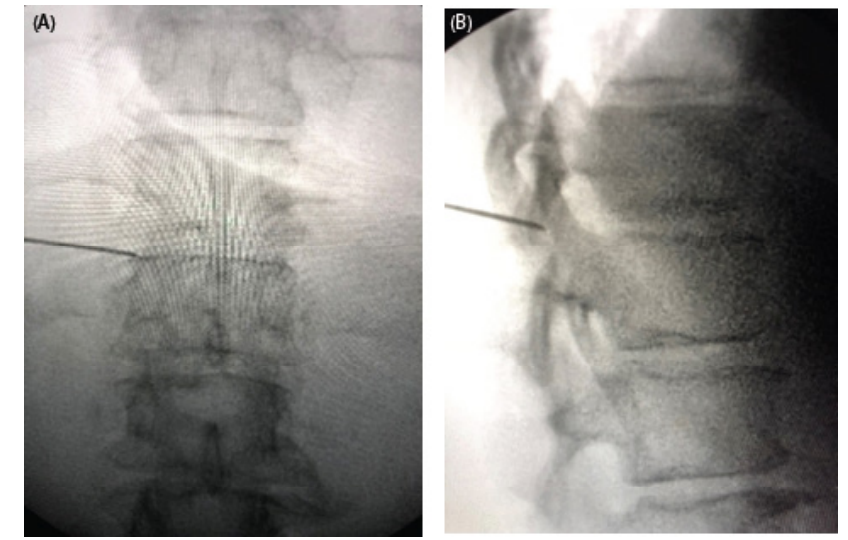

Figures 2A and 2B: Intraoperative fluoroscopy images showing the needle placement for T11/T12 disc herniation.
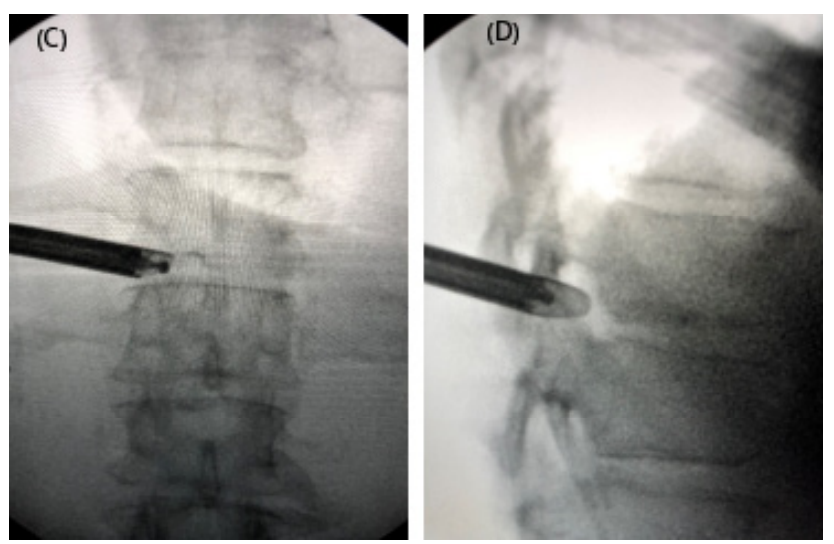

Figures 2C and 2D: Intraoperative fluoroscopy images showing cannula placement, endoscope, and high-speed drill for foraminoplasty.

as well as partially the rib head and the pedicle if needed. The precise extent of the foraminoplasty depending on the location and size of the disc herniation. The facet, pedicle, disc and ligamentum flavum were identified and visualized, and the herniated disc material was carefully removed without any manipulation or retraction on the spinal cord (Figures 3C and 3D). If the disc herniation is calcified or there is ventral endplate osteophyte, they are drilled under direct visualization with the high-speed drill. Free floating thecal sac with good pulsation is a sign of adequate decompression. Hemostasis was achieved with a radiofrequency probe. The instruments are withdrawn and the incision $(8 \mathrm{~mm})$ is closed without a drain.

\section{Results}

16 consecutive patients ( 10 females, 6 males, average age 55, range 28 to 78 ) were included in this case series. The patients were decompressed in the fully endoscopic transforaminal retropleural technique as described above. The locations of the disc herniations were T5/T6 ( 1 case), T6/T7 ( 1 case), T7/T8 (4 cases), T8/T9 ( 2 cases), T9/T10 ( 2 cases), T10/T11 ( 1 case), T11/T12 ( 5 cases). There were 7 soft and 9 calcified disc herniations, including a calcified T8/9 giant central disc herniation. The leading symptoms were radiculopathy in 13 cases and myelopathy in 3 cases. All but one patient had significant thoracic axial pain.
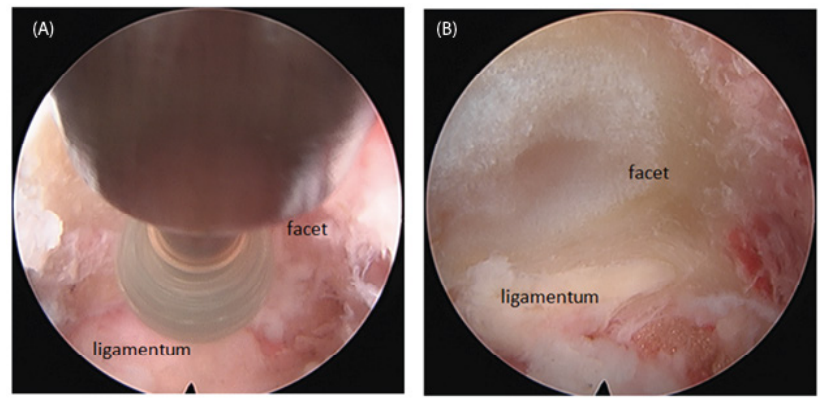

Figures 3A and 3B: Introperative endoscopic image showing drilling of the T12 superior facet, and ligamentum flavum, epidural fat after foraminoplasty.
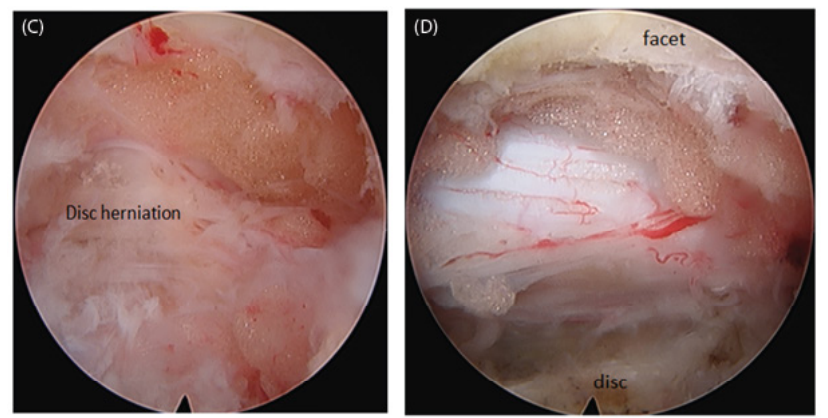

Figures 3C and 3D: Introperative endoscopic image showing the T11/T12 paracentral disc herniation, and thecal sac after discectomy.

The average operation time was approximately 90 minutes (55-180 minutes), average hospital stay was 0.3 days. There was no intraoperative complication.

That one patient with a chronic calcified T8/9 giant central disc herniation and significant myelopathy had complaints of worsening symptoms post-operatively; she subsequently underwent open multilevel laminectomy decompression and instrumented spinal fusion surgery in a different institution. Among the 15 remaining patients in this case series, the average preoperative visual analog scale score was 7.9 , the average postoperative visual analog scale score was 2.1 at final follow-up (average 21 months, range 7-60 months). No surgery or approach related increase in thoracic back pain was reported. There was no recurrent disc herniation in this series.

\section{Discussion}

The blood supply to the spine is based on a predictable segmental vascular structure at each spinal level, but true radiculomedullary arteries, which feed the dominant cord supply vessel, the anterior spinal artery, are relatively few and their locations variable [7]. The thoracic spinal cord is especially vulnerable during spinal deformity surgery or other open surgeries, sacrifice of a dominant radiculomedullary vessel may lead to spinal cord ischemia. In addition, manipulation or retraction of spinal cord must be avoided during surgery. The fully endoscopic transforaminal/retropleural technique described here, combined with angled field of view, and freedom of movement of the endoscope using a joystick technique offers adequate decompression. In addition, clear visualization and significantly reduced bleeding due to continuous irrigation, low complication rates, as well as being done under local anesthesia are unique advantages of this fully endoscopic transforaminal/retropleural approach for thoracic disc herniations. 
During the endoscopic approach, bone resection of the facet joint/ foaminoplasty is necessary for access to the spinal canal. The endoscopic foraminoplasty with high-speed drill under direct visualization offers additional safety.

One specific intraoperative complication can be dura tear, which cannot be sutured in this fully endoscopic approach, but can be covered with a synthetic dura substitute [5].

\section{Conclusion}

Fully endoscopic transforaminal/retropleaural foraminoplasty and discectomy is a safe and effective treatment option for symptomatic soft or calcified thoracic disc herniations.

\section{References}

1. Stillerman CB, Chen TC, Couldwell WT, Zhang W, Weiss MH (1998) Experience in the surgical management of 82 symptomatic herniated thoracic discs and review of the literature. J Neurosurg 88: 623-633.
2. Yoshihara $\mathrm{H}$ (2014) Surgical treatment for thoracic disc herniation: an update. Spine (Phila Pa 1976) 39: E406-12.

3. Wait SD, Fox DJ Jr, Kenny KJ, Dickman CA (2012) Thoracoscopic resection of symptomatic herniated thoracic discs: clinical results in 121 patients. Spine (Phila Pa 1976) 37: 5-40.

4. Moran C, Ali Z, McEvoy L, Bolger C (2012) Mini-open retropleural transthoracic approach for the treatment of giant thoracic disc herniation. Spine (Phila $\mathrm{Pa}$ 1976) 37: E1079-84

5. Ruetten S, Hahn P, Oezdemir S, Baraliakos X, Godolias G, et al. (2018) Operation of Soft or calcified thoracic disc herniations in the full-endoscopic uniportal extraforaminal technique. Pain Physician 21: E331-E340.

6. Wagner R, Telfeian AE, Iprenburg M, Krzok G, Gokaslan Z, et al. (2016) Transforaminal endoscopic foraminoplasty and discectomy for the treatment of a thoracic disc herniation. World Neurosurg 90: 194-198.

7. Colman MW, Hornicek FJ, Schwab JH (2015) Spinal cord blood supply and its surgical implications. J Am Acad Orthop Surg 23: 581-591.
This article was originally published in a special issue, Minimally Invasive Spine Surgery -II handled by Editor(s). Dr. Anthony T.Yeung, Desert Institute for Spine Care, USA 